\title{
DINÂMICA DA ALIMENTAÇÃO E DEJEÇÃO DO TRIATOMA SORDIDA
}

\author{
Liléia Diotaiuti, Claysson M. Penido, Herton \\ Helder R. Pires e João Carlos P. Dias
}

\begin{abstract}
Atualmente, o Triatoma sordida é a espécie de triatomineo mais capturada no Brasil. Para maior esclarecimento do seu potencial vetorial fot estudada a sua dinamica de alimentação e dejeções, em comparação com o Triatoma infestans ou outras espécies de reconbecida importancia na transmissão do Trypanosoma cruzi. O nümero de T. sordida $e \mathrm{~T}$. infestans alimentados em ratos anestesiados não foi significativamente diferente, nem a média de tempo gasta entre a liberação dos triatomineos e o inicio do repasto sanguineo. A média de tempo para eliminação da primeira dejeção foi significativamente menor em $\mathrm{T}$. infestans que em $\mathrm{T}$. sordida; no entanto, o nümero de insetos que defecou durante ou imediatamente após o repasto foi semelhante nas duas espécies. Observaçôes diârias ao longo de um mês indicam que o T. sordida realiza um maior número de picadas por dia do que o T. infestans ou o P. megistus. Estes dados sugerem alguma dificuldade desta espécle em concluir o seu repasto, fato provavelmente importante no seu processo de adaptação ao bospedeiro.
\end{abstract}

Palavras-chaves: Triatoma sordida. Dinâmica alimentar. Dejeçoes. Potencial vetorial.

Após o controle do Triatoma infestans, o Triatoma sordida tornou-se a espécie de triatomíneo mais capturada no Estado de Minas Gerais, especialmente no peridomicílió. Dentre as características que definem um bom vetor do Trypanosoma cruzi inclui-se o comportamento alimentar, o ritmo de dejeções do triatomíneo e o número de picadas realizadas durante o processo alimentar, trazendo potencialmente mais risco de transmissão aqueles que apresentem maior possibilidade de contato com o hospedeiro vertebrado, e menor tempo para eliminação das dejeções, de forma que as mesmas sejam depositadas ainda sobre o hospedeiro ${ }^{2}$.

Considerando-se a freqüencia com que o $T$. sordida tem sido capturado no ambiente artificial, para contribuir com o esclarecimento do seu potencial vetorial realizaram-se alguns experimentos sobre a sua dinâmica de alimentação e dejeção, em comparação com o T. infestans e o Panstrongylus megistus,

Centro de Pesquisas René Rachou, Fundação Oswaldo Cruz, e Departamento de Parasitologia do Instituto de Ciências Biológicas da Universidade Federal de Minas Gerais, Belo Horizonte, MG.

Endereço para correspondência: Dra. Liléia Diotaiuti. Centro de Pesquisas René Rachou. Caixa Postal 1743, 30190-002 Belo Horizonte, MG.

Recebido para publicação em 12/08/94. espécies de comprovada importância epidemiológica.

\section{MATERIAL E MÉTODOS}

Todos os experimentos foram realizados com lotes de insetos da mesma idade (mesmo estádio, e ecdise processada no mesmo dia), alimentados em aves há quinze dias. Utilizaram-se ninfas de primeiro estádio, sete dias após a eclosão dos ovos e em completo jejum.

Dinâmica da alimentação e dejeções: Foram alimentadas em camundongos normais 25 ninfas de quarto estádio de $T$. sordida. O repasto e as dejeções foram acompanhados individualmente por duas horas, em dispositivo Ryckman modificado por Bronfen, Dias \& Rocha, $1980^{1}$ (Figura 1). A temperatura no ambiente foi de $28 \pm 1^{\circ} \mathrm{C}$, com $68 \pm 3 \%$ de umidade relativa do ar.

O tempo gasto entre a oferta de alimento e início do repasto foi medido em outro grupo homogêneo, constituido por 30 ninfas de terceiro estádio. Os insetos foram soltos simultaneamente em duas bandejas (uma para cada espécie), contendo, cada uma, um rato anestesiado posicionado no lado oposto àquele onde os insetos foram colocados. Anotava-se o tempo que cada inseto gastava para encontrar a fonte alimentar e iniciar o 
Diotaiuti L, Penido CM, Pires HHR, Dias JCP. Dinâmica da alimentação e dejeção do Triatoma sordida. Revista da Sociedade Brasileira de Medicina Tropical 28:195-198, jul-set, 1995.

processo de alimentação, quando o mesmo era retirado do experimento. As observações restringiram-se a um período de 45 minutos, tendo se realizado às 12 horas, em local com iluminação natural, e às 19 horas, com luz fraca, artificial e indireta. As medidas de temperatura e umidade tomadas no início e no final dos experimentos não foram estatisticamente diferentes (média de temperatura $=27 \pm 2^{\circ} \mathrm{C}$; média de umidade de $64 \pm 2 \%$ ). Paralelamente foi estudado o $T$. infestans, para fins de comparação.

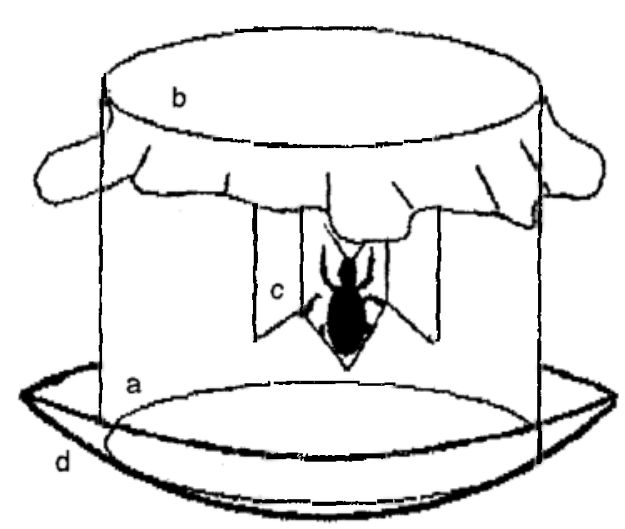

Figura 1 - Dispositivo utllizado para acompanbamento do processo de altmentação e dejeção dos triatomineos: a) frasco de borel recoberto com b) malha de nylon onde se fixa c) sanfona de papel utilizada como suporte pelo triatomineo; d) vidro de relógio utilizado para receber as dejeçōes.

Número de picadas diárias: foi estimado o número médio de picadas que um grupo de cerca de 80 triatomíneos adultos é capaz de realizar ao longo de quatro semanas consecutivas, em observaçôes diárias de 30 minutos. Os insetos eram soltos em uma bandeja onde havia um rato anestesiado, e o número de picadas realizadas era contado. Os resultados referentes ao $T$. sordida foram comparados aos também obtidos com $T$. infestans e $P$. megistus.

\section{RESULTADOS}

Na Tabela 1 são apresentados os resultados referentes ao estudo da dinâmica alimentat e das dejeções do $T$. sordida e do $T$. infestans. Vinte e uma (84\%) ninfas de $T$. sordida e 20 $(80 \%)$ de T. infestans $(p=0,5)$ alimentaram-se, com tempos médios para a completa repleção de 29'4" $\pm 19^{\prime} 8^{\prime \prime}$ e 36'40" $\pm 22^{\prime} 57^{\prime \prime},(0,2<\mathrm{p}<$ $0,3)$, respectivamente. A média de tempo entre a eliminação da primeira dejeção pelo $T$. infestans (36' $\pm 26^{\prime}$ ) foi significativamente menor que a média correspondente ao $T$. sordida $\left(51^{\prime} \pm 30^{\prime}\right)$; por outro lado, o número de triatomíneos que defecou durante a realização do repasto foi estatisticamente semelhante entre as duas espécies (8 exemplares de $T$. sordida e 6 de $T$. infestans, $\mathrm{p}=0,7)$, assim como o número de insetos que defecaram imediatamente após o repasto (10 $T$. sordida e $12 T$. infestans; $\mathrm{p}=0,7)$.

Tabela 1- Dinâmica da alimentacão e dejeçães de T. sordida $e \mathrm{~T}$. infestans acompanbados por duas boras em camundongos anestesiados.

\begin{tabular}{lrrr}
\hline Observações & T, sordida & T. inffestans & \multicolumn{1}{c}{$\mathrm{p}$} \\
\hline $\begin{array}{l}\text { No insetos que } \\
\text { se alimentaram }\end{array}$ & $21(84 \%)$ & $20(80 \%)$ & 0,5 \\
Tempo para repleção & $29^{\prime} 4^{\prime \prime} \pm 19^{\prime} 8^{\prime \prime}$ & $36^{\prime} 0^{\prime \prime} \pm 22^{\prime} 57^{\prime \prime}$ & $>0,05$ \\
Tempo para 1a dejeção & $51^{\prime} \pm 30^{\prime \prime}$ & $36^{\prime} \pm 26^{\prime \prime}$ & $<0,05$ \\
$\begin{array}{l}N^{2} \text { insetos que defecaram } \\
\text { durante o repasto }\end{array}$ & 8 & 6 & 0,7 \\
$\begin{array}{l}N^{2} \text { insetos que defecaram } \\
\text { imediatamente após o repasto }\end{array}$ & 10 & 12 & 0,7 \\
\hline $\mathrm{n}=25$ triatomíneos por espécie. & & & \\
\hline
\end{tabular}

Na Tabela 2 podem-se observar os resultados obtidos na experiência que mediu a atração do $T$. sordida e do $T$. infestans pela fonte de alimentação. $O$ número de exemplares de $T$. sordida que iniciou $\circ$ processo de alimentação no experimento diurno $(90 \%)$ foi maior que no experimento noturno $(50 \%)(p=0,001)$. Esta comparação entre $T$. sordida e $T$. infestans/manhã $(90$ e $86,7 \%$, respectivamente; $\mathrm{p}=0,5)$ e noite $(50 \mathrm{e}$ $66,7 \%$, respectivamente; $\mathrm{p}=0,2$ ) não revelou diferença estatística. Em relação ao tempo gasto entre a oferta do alimento e início do repasto, também não houve diferença entre as duas espécies ( $p>0,05)$.

Tabela 2- Temoo médio gasto por ninfas de terceiro estádio de $\mathrm{T}$. sordida $e \mathrm{~T}$. infestans entre a liberaça das mesmas e o início do repasto, em presença de luz natural (manbã)ou na penumbra (tarde).

\begin{tabular}{|c|c|c|c|}
\hline Horário & $\begin{array}{l}\text { Espécie de } \\
\text { triatomineo }\end{array}$ & $\begin{array}{l}\mathrm{N}^{\mathrm{O}} \text { de insetos } \\
\text { que se alimentatam }\end{array}$ & $\begin{array}{l}\text { Tempo médio até } \\
\text { o início do repasto }\end{array}$ \\
\hline \multirow[t]{2}{*}{ Manhā } & T. sordida & $27 \quad(90 \%)$ & $13^{\prime} 19^{\prime \prime} \pm 12^{\prime} 02^{\prime \prime}$ \\
\hline & T. infestans & $(86,7 \%)$ & $9^{\prime} 05^{\prime \prime} \pm 10^{\prime} 12^{\prime \prime}$ \\
\hline \multirow[t]{2}{*}{ Tarde } & T. sordida & $(50 \%)$ & $14^{\prime} \pm 8^{\prime} 37^{\prime \prime}$ \\
\hline & T. infestans & $(66,7 \%)$ & $18^{\prime} 05^{\prime \prime} \pm 10^{\prime} 20^{\prime \prime}$ \\
\hline
\end{tabular}

No estudo do número de picadas/triatomíneo/dia (Tabela 3), realizado através da análise de variância, o T. sordida realizou maior número de picadas $(0,8 \pm 0,3)$, em comparação ao $T$. infestans $(0,5 \pm 0,3)$ e $P$ megistus $(0,4 \pm 0.3)(\mathrm{p}<0,05)$. 
Diotaiuti L, Penido CM, Pires HHR, Dias JCP. Dinâmica da alimentação e dejeção do Triatoma sordida. Revista da Sociedade Brasileira de Medicina Tropical 28:195-198, jul-set, 1995.

Tabela 3- Nümero médio semanal de picadas realizadas por $\mathrm{T}$. sordida, $\mathrm{T}$. infestans $e \mathrm{P}$. megistus colocados em contato com rato anestesiado por 30 minutos, diariamente, por quatro semanas consecutivas.

\begin{tabular}{lccc}
\hline Semana & T. sordida & T. infestans & P. megistus \\
\hline $1^{\mathrm{a}}$ & $0,7 \pm 0,5$ & $1,0 \pm 0,4$ & $0,6 \pm 0,5$ \\
$2^{\mathrm{a}}$ & $0,3 \pm 0,1$ & $0,7 \pm 0,4$ & $0,3 \pm 0,2$ \\
$3^{\mathrm{a}}$ & $0,5 \pm 0,1$ & $0,8 \pm 0,2$ & $0,5 \pm 0,2$ \\
$4^{\mathbf{a}}$ & $0,5 \pm 0,2$ & $0,6 \pm 0,3$ & $0,4 \pm 0,1$ \\
\hline Total & $0,5 \pm 0,3$ & $0,8 \pm 0,3$ & $0,4 \pm 0,3$ \\
\hline
\end{tabular}

\section{DISCUSSÃO}

A voracidade de um triatomíneo é a medida da rapidez com que o inseto inicia o repasto sanguíneo e o complementa. A maioria dos dados disponíveis sobre este tema refere-se à resposta dos insetos (início da alimentação) à oferta de alimento. Em estudo comparativo entre o $T$. infestans, $T$. sordida, Triatoma brasiliensis, Triatoma pseudomaculata, Panstrongylus megistus e Rhodnius neglectus?, apenas as ninfas de primeiro estádio recém eclodidas de $T$. sordida $(4,4 \%$ da amostra estudada) e $P$. megistus (3,1\%) foram capazes de se alimentar no primeiro dia após a eclosão dos ovos. No segundo dia, $95,8 \%$ das ninfas de $T$. sordida responderam ao estímulo alimentar, e no terceiro todas estavam aptas a fazê-lo, o que aconteceu com o $T$. infestans no quarto dia e com o $P$. megistus no quinto dia. Em relação ao $T$. sordida, Mello $(1980)^{6}$ obteve resposta de todas as ninfas de primeiro estádio ao estímulo alimentar tão rapidamente quanto o T. infestans.

A maior facilidade para alimentação no experimento diurno, em ambas as espécies, está em discordância com os sabidos hábitos noturnos de algumas espécies de triatomíneos, em especial o $T$. infestans 5 . O fato parece indicar inversão do comportamento dos insetos em insetário, mantidos no escuro a maior parte do tempo, exceto no momento da alimentação, realizada em local com luminosidade.

Dias (1956)² chamava a atenção para o diferente comportamento dos triatomíneos em relação à dinâmica das dejeções, identificando os de maior potencial vetorial entre aqueles que apresentam maior frequência na eliminação das dejeções, os que defecam mais vezes durante ou logo após o répasto sanguíneo, os que completam a alimentação em menor tempo e apresentam maior inclinação para interromper a picada. As duas últimas características estariam relacionadas à maior proteção do inseto contra reações de agressão pelo hospedeiro. Por estes critérios, o $R$. prolixus foi considerado o triatomíneo com maior potencial vetorial, seguido do $R$. neglectus, $T$. infestans e $P$. megistus, não merecendo o $\mathrm{T}$. sordida maiores comentários por parte do autor, apesar dos resultados obtidos para esta espécie não terem sido muito diferentes dos apresentados pelo P. megistus, espécie considerada de grande importância epidemiológica. No presente experimento, apesar da primeira dejeção ter sido eliminada mais rapidamente pelo $T$. infestans, os demais parâmetros medidos (número de triatomíneos que se alimentaram, tempo para repleção, número de triatomíneos que defecou durante ou imediatamente após o repasto) também não foram desfavoráveis ao $T$. sordida.

Para Rabinovich e cols ${ }^{7}$ a agressividade que um triatomíneo apresenta para obtenção do seu repasto, medida por eles através da chamada "biting rate", pode representar, por um lado, um maior contato do triatomíneo com o hospedeiro, servindo como parâmetro de medida de potencial vetorial. Por outro lado, a rapidez com que o inseto completa seu repasto, ou seja, sua capacidade intrínseca de sucção aliada ao menor número de interrupções neste processo (com menor número de picadas subseqüentes), aumenta a sua possibilidade de sobrevivência, tanto sob o ponto de vista da sua proteção contra reações do hospedeiro quanto pela possibilidade de obtenção do sangue necessário para o processamento da muda para o próximo estádio com apenas um repasto ${ }^{7}$. O maior número médio de picadas realizadas pelo $T$. sordida em relação às demais espécies estudadas observado no nosso experimento sugere agressividade da espécie no seu processo alimentar, e um bom "contact feeding time", o que o habilitaria por Dias ${ }^{2}$ como potencial vetor domiciliar do $T$. cruzi. No entanto, as experiências no campo ${ }^{4} 810$ demonstram que, apesar da espécie colonizar com facilidade os ecótopos peridomiciliares, onde a principal fonte alimentar são as galinhas, o T. sordida apresenta dificuldades para formar grandes colônias intradomiciliares, a exemplo do Triatoma vitticeps ${ }^{3}$. Esta limitação poderá, possivelmente, estar relacionada à dificuldade de obtenção de sangue humano pelo triatomíneo, principal fonte de alimentação no intradomicílio, e não 
Diotaiuti L, Penido CM, Pires HHR, Dias JCP. Dinâmica da alimentação e dejeção do Triatoma sordida. Revista da Sociedade Brasileira de Medicina Tropical 28:195-198, jul-set, 1995.

somente aos parâmetros aqui estudados, hipótese que merece ser futuramente investigada na expectativa de esclarecer quais são os verdadeiros fatores que determinam a adaptação do triatomíneo às suas fontes alimentares.

\section{SUMMARY}

Today, Triatoma sordida is the most frequently captured triatomine in Brazil. For a better understending of its vectorial potential, its feeding dynamics and dejections behaviour were studied in comparison with Triatoma infestans and $\mathrm{P}$. megistus. The proportion of $\mathrm{T}$. sordida and $\mathrm{T}$. infestans that blood fed on anaesthetized rats did not differ significantly. There was no significant difference in the time elapse between release of the bugs and the initiation of blood feeding. The mean time between completion of a blood meal and the first dejection was significantly lower in $\mathrm{T}$. infestans than in $\mathrm{T}$. sordida. The numbers of insects that defecated during the blood meal or immediatly afterwards was similar for botb species. Approximately 80 specimens of each of $\mathrm{T}$. sordida, $\mathrm{T}$. infestans and Panstrongylus megistus were offered daily blood meals for 30 minuts, over a period of 30 days. $\mathrm{T}$. sordida bit more frequently than did $\mathrm{P}$. megistus and $\mathrm{T}$. infestans. These data could indicate that $\mathrm{T}$. sordida may not be completely adapted to its bost and might bave difficulty in complete its blood meal.

Key-words: Triatoma sordida. Blood feeding dynamics. Defecation behaviour. Vectorial potential.

\section{AGRADECIMENTO}

Ao Prof. Paul Williams, pela revisão do resumo em inglês.

\section{REFERÊNCIAS BIBLIOGRÁFICAS}

1. Bronfen E, Dias JCP, Rocha FSA. Studies on the spontaneous elimination of infectant dejections of Triatoma infestans infected with Trypanosoma cruzi ( $Y$ strains).In: Resumos da VII Reunião Anual Pesquisa Básica Doença Chagas, Caxambu, V21, 1980.

2. Dias E. Observação sobre eliminação de dejeções e tempo de sucção em alguns triatomíneos sul- americanos. Memórias do Instituto Oswaldo Cruz 54:115-124, 1956.

3. Dias JCP, Feitosa VR, Ferraz Filho A, Rodrigues VLC, Alencar SA, Sessa PA. Fonte alimentar e potencial vetorial do Triatoma vitticeps com relação a doença de Chagas humana no Estado do Espírito Santo, Brasil (Hemiptera, Reduviidae). Memórias do Instituto Oswaldo Cruz 84:165-173, 1989.

4. Diotaiuti L. Importância atual e perspectivas de controle do Triatoma sordida em Minas Gerais, Brasil.Tese de doutorado, Universidade Federal de Minas Gerais, Belo Horizonte, MG, 1991.

5. Espínola HN. Aspectos do comportamento do Triatoma infestans (Klug, 1834) em condiçöes experimentais de laboratório (Hemiptera, Reduviidae, Triatominae). Tese de doutorado, Universidade Federal de Minas Gerais, Belo Horizonte, MG, 1973.

6. Mello DA. Some aspects of feeding habits under laboratory conditions of Triatoma sordida (Stal, 1859) and Rhodnius neglectus Lent, 1954 (Hemiptera, Reduviidae). Revista Brasileira Biologia 40:323-326, 1980.

7. Rabinovich JE, Leal JA, Feliciangelli de Piñero D. Domiciliary biting frequency and blood ingestion of the Chagas's disease vector Rhodnius prolixus Stahl (Hemiptera: Reduviidae), in Venezuela. Transaction of the Royal Society of Tropical Medicine and Hygiene 73:272-283, 1979.

8. Souza AG, Wanderley DMV, Buralli GM, Andrade JCR. Consolidation of the control of Chagas disease vectors in the state of São Paulo. Memórias do Instituto Oswaldo Cruz 79:125-131, 1984.

9. Szumlewicz AP. Laboratory colonies of triatominae, biology and population dynamics. In: New Approaches in American Trypanosomiasis Research p.63-82, Belo Horizonte, Brazil, 1975.

10. Zapata MTG. Controle da doença de Chagas com inseticidas e participação comunitária $\mathrm{em}$ Mambai-Go. Tese de Doutorado, Universidade Federal de Minas Gerais . Belo Horizonte, MG, 1990. 